\title{
Control of Distributed Servers for Quality-Fair Delivery of Multiple Video Streams
}

\author{
Nesrine Changuel, Bessem Sayadi Alcatel-Lucent - Bell-Labs France, \\ Route de Villejust, F-91620 Nozay Michel Kieffer L2S, CNRS - SUPELEC - Univ Paris-Sud, F-91192 \\ Gif-sur-Yvette \\ LTCI, CNRS - Télécom ParisTech, F-75013 Paris
}

\begin{abstract}
This paper proposes a quality-fair video delivery system able to transmit several encoded video streams to mobile users sharing some wireless resource. Video quality fairness, as well as similar delivery delay is targeted among streams. The proposed control system is implemented within some aggregator located near the bottleneck of the network.

This is done by allocating the transmission rate among streams based on the quality of the already encoded and buffered packets in the aggregator. Encoding rate targets are evaluated by the aggregator and fed back to each remote video server, or directly evaluated by each server in a distributed way. Each encoding rate target is adjusted for each stream independently based on the corresponding buffering delay in the aggregator.

The transmission and encoding rate control problems are addressed with a control-theoretic perspective. The system is described with a multi-input multi-output model and several Proportional Integral (PI) controllers are used to adjust the video quality as well as the buffering delay. The study of the system equilibrium and stability provides guidelines for choosing the parameters of the PI controllers. Experimental results show that better quality fairness is obtained compared to classical transmission rate fair streaming solutions while keeping similar buffering delays.
\end{abstract}

\section{Index Terms}

Video, Control design, Distributed systems

\section{INTRODUCTION}

Traffic corresponding to compressed videos is increasing rapidly, especially to mobile users, which are consuming more and more video-on-demand or mobile TV broadcast services. This trend is likely to continue in the coming years [1]. Streaming or broadcasting videos to several users sharing a bandlimited wireless channel with timevarying characteristics is still a challenging task. The demand for better video quality with limited transmission delays are the main problems to be addressed.

Increasing the rate at which videos are encoded improves their quality, but the price to be paid is an increased congestion at the bottleneck of the network, usually lying at the interface of the wired and wireless part, when considering video delivery to mobile users. To maximize the overall quality of the delivered video streams, it is thus preferable, instead of equally dividing the available resource, e.g., bandwidth or transmission rate, among streams or among users, to have resource allocation and video server control algorithms achieving a fair video quality among streams to deliver streams with comparable (objective or subjective) quality and delivery delay.

This paper proposes a quality-fair video transmission system able to deliver several encoded streams to mobile users over some bottleneck link. Typical application contexts are mobile broadcast or parallel unicast of video contents to users connected to some base station. Video encoding rate adaptation and wireless resource allocation are performed jointly within some Media Aware Network Element (MANE) using feedback control loops. The aim is to provide users with encoded videos of similar quality and with controlled delivery delay, without exchanging information between remotely located video servers.

\section{A. Related works}

When controlling the multiple video streams, their rate-distortion (R-D) trade-off may be adjusted by selectively discarding frames as in [2], [3] or via an adjustment of the encoding parameters [4], [5]. With scalable video 
encoders, such as H.264/SVC, the R-D trade-off may be adjusted via packet filtering [6], [7]. In this case, the number of transmitted enhancement layers for each frame is the control parameter.

Video quality fairness among encoded streams may be obtained by sharing quality information, or R-D characteristics via a central controller providing to each server quality or rate targets [8], [9]. This technique enables the encoders to adjust their rate or to drop frames depending on the complexity of the videos and on the available transmission rate.

Control-theoretic approaches to address the video streaming rate control problem have been considered, e.g., in [10], [11], [12], [13], and [14]. In [10], a real-time rate control based on a Proportional Integral Derivative (PID) controller is proposed for a single video stream. The main idea is to determine the encoding rate per frame based on the buffer level to maximize video quality and minimize quality variations over frames. In [11], a flow control mechanism with active queue management and a proportional controller is considered. A flow control is used to reduce the buffer size and avoid buffer overflow and underflow. Stability issues are addressed to show that the flow control mechanism is stable for small buffer sizes and non-negligible round-trip times. In [12], the rate allocation algorithm, performed at the Group of Picture (GoP) level, is in charge of determining the transmission rate from the buffers and a playback adjustment mechanism. A Proportional Integral (PI) controller is adopted at the receiver to maximize the visual quality according to the overall loss and the receiver buffer occupancy.

Later, in [15], a rate control that uses different bit allocation strategies for intra and inter frames is introduced. A PID controller is adopted to minimize the deviation between the target buffer level and the current buffer fullness. However, buffer management is performed at the bit level and delivery delay is not considered. Moreover, [10], [11], [12], [15] address single flow transmission.

For multi-video streaming, in [16], a distributed utility max-min flow control in the presence of round-trip delays is proposed. The distributed link algorithm attains utility max-min bandwidth sharing while controlling link buffer occupancy to a target value at the cost of link under utilization using a PID controller. Stability analysis in case of a single bottleneck and a homogeneous delay is conducted.

In [8], a content-aware distortion-fair video delivery scheme is proposed to deliver video streams based on the characteristics of video frames. It provides a max-min distortion fair resource sharing among streams. The system uses temporal prediction structure of the video sequences with a frame drop distortion metric based on the frame importance to guide resource allocation. This scheme is for stored video, where the side information of the number of bits and importance of the frames is assumed calculated in advance.

A proportional controller is considered in [13] to stabilize the received video quality as well as the bottleneck link queue for both homogeneous and heterogeneous video systems. A PI controller is considered in [14]. Robustness and stability properties are studied. In [13] and [14], a centralized rate control exploits the rate and distortion characteristics of the transmitted video streams to determine the encoding rate for the next frames of each session.

The problem of remotely implemented control law is also considered in [17] leading to the problem of stabilizing an open-loop unstable system with time-varying delay. The problem of remote stabilization via communication networks is considered with an explicit use of the average network dynamics and an estimation of the average delay dynamics in the control law. The control law does not address video transmission issues, so no quality constraint on the transmitted data is considered.

The commercial product described in [18], [19] propose statistical multiplexing systems enabling encoders to adapt their outputs to the available channel rate. Connecting encoders and multiplexers via a switched IP network allows collocated as well as distributed encoders to be part of the multiplexing system. Nevertheless, in these solutions, the rate control process is centralized and quality fairness constraints between programs appear not to be considered among the video quality constraints.

\section{B. Main contributions}

In this paper, we propose a partially distributed control system to perform jointly $(i)$ encoding rate control of spatially spread video servers without requiring exchange of information between them and (ii) transmission rate control of the encoded streams through some bottelneck link. A MANE, located near the bottelneck link contains dedicated buffers fed by the remote servers. It performs a centralized transmission rate control for each encoded video stream, adjusting the draining rate of each buffer. For that purpose, it uses quality information of the encoded videos to favor programs with lower quality. Dedicated encoding rate controllers observe the buffering 
Media Aware Network Element

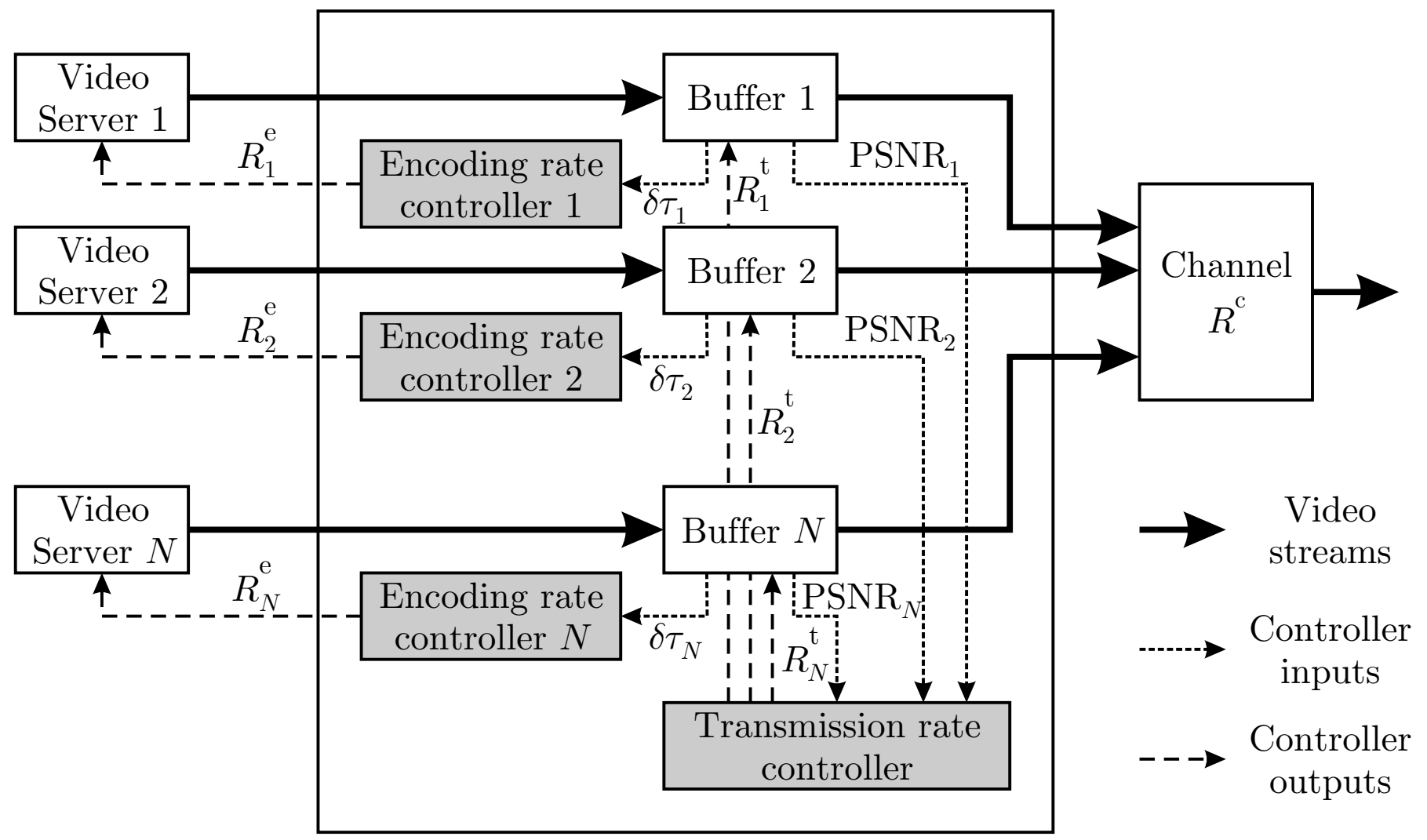

Fig. 1. Structure of the proposed quality-fair video delivery system (fully centralized version).

delay discrepancy with respect to some reference to adjust the encoding rates. In a fully centralized solution, the MANE is then in charge of sending the encoding rate target to each video server. In a partly distributed solution, only the individual delay discrepancies are transmitted to the servers, which are then in charge of computing their encoding rate target. The proposed multi-input, multi-output (MIMO) closed-loop feedback control system has to regulate the buffering delay and to provide quality fairness between video streams. For that purpose, the feedback loops involve PI controllers which stability is studied. Quality fairness constraint among streams results in a coupling of the state equation related to each stream. The stability analysis of the coupled state equations is a challenging task which was not performed previously.

Section II describes the considered system and the constraints that have to be satisfied. The proposed solution is described in Section III. A discrete-time state-space representation of the MIMO system is provided, emphasizing the coupling induced by the fairness constraint among video streams. The equilibrium and stability analysis are performed in Section IV. This provides some insight on the way the various PI controllers should be tuned. Then, a typical application context is described in Section V. Finally, experimental results are detailed in Section VI, showing that the proposed control system allows transmitting several video programs in parallel with comparable video quality and similar buffer delay.

\section{SYSTEM DESCRIPTION}

Consider a communication system in which $N$ encoded video streams provided by remote servers arrive to some network bottelneck where they have to share a communication channel providing a transmission rate $R^{\mathrm{c}}$, see Figure 1. Encoded Video Units (VUs) representing a single picture or a Group of Pictures (GoP) are provided by the servers. All VUs are assumed to be of the same duration $T$ and the frame rate $F=1 / T$ is assumed constant over time and identical for all streams. At time index $j$, all servers encode, transcode, or layer filter (in case of scalable video streams) the $j$-th VU. 
We assume that a Media Aware Network Element (MANE) is located close to the bottelneck of the network, see Figure 1. The aim of the MANE is to allocate the available transmission rate $R^{\mathrm{c}}$ among video streams in such a way that they are delivered with similar reconstruction quality (objective or subjective), and with similar delivery delay, to get a quality-fair video delivery system.

For that purpose, encoded and packetized VUs are temporarily stored in dedicated buffers in the MANE. Moreover, two feedback loops are considered to control the encoding and transmission rate of each stream, see Figure 2. In a fully centralized version, each individual encoding rate target is evaluated in the MANE and fed back to each server as shown in Figure 1. In a partly decentralized version, each encoding rate is evaluated by the remote video servers. In both cases, the transmission rates evaluated in a centralized way within the MANE determine the draining of the buffers in the MANE. This control is performed at discrete time instants, with a period $T$ equal to the duration of a VU.

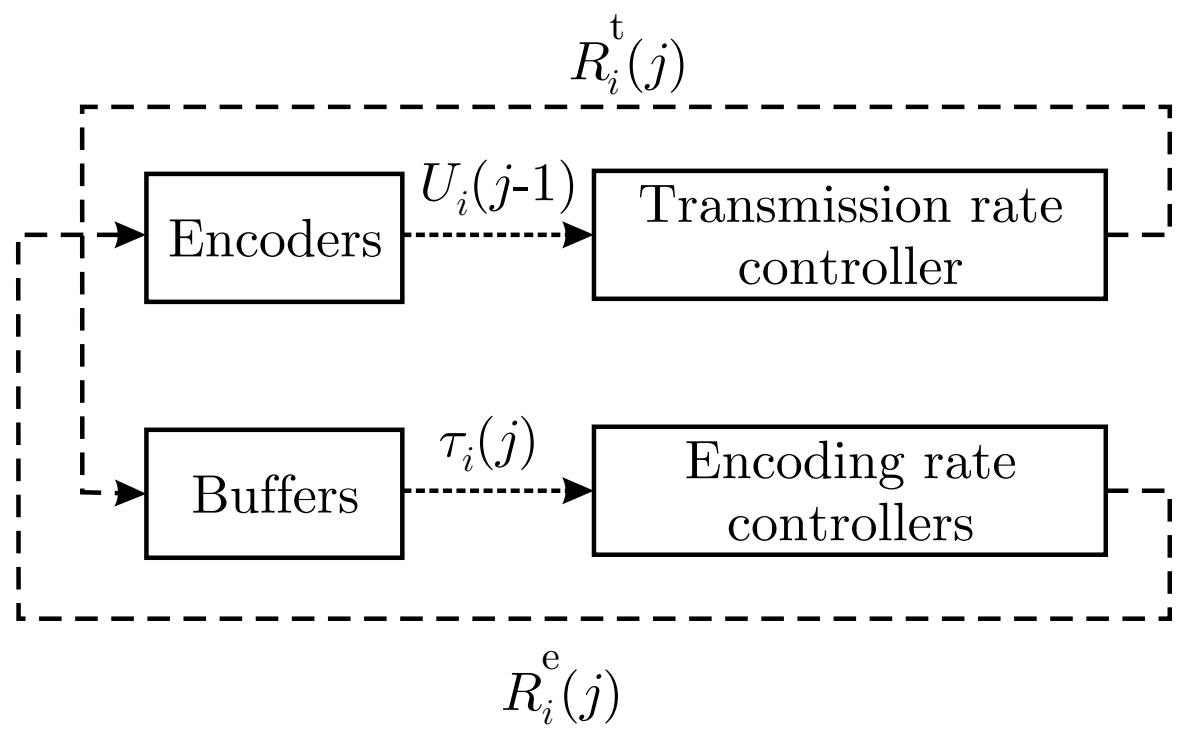

Fig. 2. Feedback control loops in the proposed quality-fair video delivery system.

A utility $U$ providing information on the video quality (PSNR, SSIM, or any other video quality metrics [20]) of the buffered video packets is assumed to be available to the MANE. The feedback loops have to be designed to minimize the discrepancy between the instantaneous utility of each video stream, and to adjust the buffering delay in the MANE around some reference delay $\tau_{0}$.

In the proposed solution, the utilities are assumed to be stored in the headers of packets containing the encoded VUs. The MANE evaluates the average utility and allocates more transmission rate to programs with a utility below average. The buffers of these programs are likely to drain faster than those with utility above average. This control, done within the MANE, is thus centralized.

The discrepancy of each buffering delay with respect to $\tau_{0}$ is processed by a dedicated encoding rate (transcoding rate or layer filtering) controller for each video stream. The encoding rate target for the next VU of each video stream is then evaluated to regulate the buffering delay around $\tau_{0}$ to prevent buffer over or underflow. The encoding rate may be evaluated at the MANE, in which case, the control is fully centralized. Here, the video encoders/servers receive only the target bit rate sent by the MANE. Alternatively, the encoding rate may be evaluated in a decentralized way at each video encoder/server. For that purpose, the MANE only feeds back to each server the buffering delay discrepancy. The latter solution requires all video encoders/servers to host individually an encoding rate controller, which is mainly possible for managed video servers. Here, the encoding rate target is evaluated within the MANE, but the encoding parameters are evaluated in a distributed way by each server [21].

The interaction of both control loops allow to get a quality-fair video delivery. Videos with a quality below average have a buffer in the MANE that is drained faster, and thus is likely to be below $\tau_{0}$. Their encoding rate target is then increased to increase their quality. In what follows, the stability of both control loops is studied. 


\section{STATE-SPACE REPRESENTATION}

The stability analysis of the control loops requires a state-space representation of the system introduced in Section II. Several additional hypotheses are needed to get a tractable representation.

\section{A. Hypotheses}

In what follows, a VU represents a GoP. All encoded VUs processed by the video servers during the $(j-1)$-th time slot $[(j-1) T, j T)$ are assumed to have reached the MANE during the $j$-th time slot $[j T,(j+1) T)$. This hypothesis allows to take into account some program-dependent and time-varying transmission delays between the servers and the MANE, as long as these delays are less than $T$. Moreover, due to these delays, for the transmission rate control during the $j$-th time slot, the MANE is assumed to have only access to the utility $U_{i}(j-1)$ of the $(j-1)$-th encoded VUs. This utilities are assumed to be stored in encoded packet headers.

Feedback delays between the MANE and the video servers are neglected. This is reasonable when the MANE only feeds back signalization to carry, encoding rate targets or buffering delay discrepancies to the servers. Provided that the network is not too congested, this takes tens of milliseconds, which is negligible compared to the duration of VUs when they represent GoPs. Thus, the encoding rate $R_{i}^{\mathrm{e}}(j)$ evaluated from the buffering delay discrepancy at the beginning of the $j$-th time slot for the $i$-th video stream is assumed available without delay at the $i$-th remote video server to encode the $j$-th VU. This assumption is more questionable when performing a control of VUs representing single frames.

1) Source model: The same parametric rate-utility model is used to describe the evolution of the utility $U_{i}(j)$ as a function of the rate $R_{i}^{\mathrm{e}}(j)$ used to encode the $j$-th VU of the $i$-th stream

$$
U_{i}(j)=f\left(\mathbf{a}_{i}(j), R_{i}^{\mathrm{e}}(j)\right) .
$$

The parameter vectors $\mathbf{a}_{i}(j) \in \mathcal{A} \subset \mathbb{R}^{N_{a}}$ is time varying and program dependent. For all values of a belonging to the set of admissible parameters $\mathcal{A}, f(\mathbf{a}, R)$ is assumed to be a continuous and strictly increasing function of $R$, with $f(\mathbf{a}, 0)=0$. The time variation of $\mathbf{a}_{i}(j)$ is described by

$$
\mathbf{a}_{i}(j+1)=\mathbf{a}_{i}(j)+\delta \mathbf{a}_{i}(j),
$$

where $\delta \mathbf{a}_{i}(j)$ represents the (uncontrolled) variations of the source characteristics.

The model (1) may represent the variation with then encoding rate of the SNR, the PSNR, the SSIM, or any strictly increasing quality metric. For Gaussian sources, (1) may be easily characterized when the utility is the PSNR in $\mathrm{dB}$, see Example 1, but extends to video streams. For that purpose, the parameter vector $\mathbf{a}_{i}(j)$ in $(1)$ has to be estimated at server side, for each VU, using, e.g., encoding trials [22], [23].

Example 1: Assume that samples belonging to the interval $[0,255]$ have been generated by a Gaussian source emitting independent symbols with mean 128 and time-varying variance $\sigma^{2}(j)$ with a period $T$. Neglecting the clipping to $[0,255]$, and considering a quadratic distortion measure, the rate-distortion function for this source is

$$
D(j)\left(R^{\mathrm{e}}(j) T\right)=\sigma^{2}(j) 2^{-2 R^{\mathrm{e}}(j) T}
$$

and assuming that the utility is the PSNR, one gets

$$
U(j)=10 \log _{10}\left(\frac{255^{2}}{\sigma^{2}(j)}\right)+6 T R^{\mathrm{e}}(j)=a^{(1)}(j)+a^{(2)}(j) R^{\mathrm{e}}(j),
$$

with $a^{(1)}(j)=10 \log _{10}\left(\frac{255^{2}}{\sigma^{2}(j)}\right)$ and $a^{(2)}(j)=6 T \mathrm{~dB} . \mathrm{s} / \mathrm{bit}$.

2) Buffer model: As introduced in Section II, the MANE contains dedicated buffers for each of the $N$ encoded video streams. The evolution of the level in bits of the $i$-th buffer between time slot $j$ and $j+1$ is

$$
B_{i}(j+1)=B_{i}(j)+\left(R_{i}^{\mathrm{e}}(j-1)-R_{i}^{\mathrm{t}}(j)\right) T,
$$

where $R_{i}^{\mathrm{t}}(j)$ is the transmission rate for the $i$-th stream and $R_{i}^{\mathrm{e}}(j-1)$ is the encoding rate of the $(j-1)$-th VU. $R_{i}^{\mathrm{e}}(j-1)$ is considered in (5) due to the time encoded packets require to reach the MANE.

This paper controls the buffering delay instead of the buffering level in bits. In delay-sensitive applications, the buffering delay plays a more important role than the buffer level in bits. Since video programs are not encoded at 
the same rate, a similar buffer level in bits may correspond to very different buffering delays. Assume that $h_{i}(j)$ is the number of VUs in the $i$-th MANE buffer at time $j$. The buffering delay of the $i$-th stream at time $j$ is $\tau_{i}(j)=h_{i}(j) T$.

Assume that packets containing encoded VUs are segmented to allow a fine granularity of the draining rate. Then $h_{i}(j)$ is difficult to evaluate within the MANE using only information stored in packet headers. The buffering delay for the $i$-th buffer is then approximatively evaluated as $\tau_{i}(j)=B_{i}(j) / \bar{R}_{i}^{\mathrm{e}}(j)$, where

$$
\begin{aligned}
\bar{R}_{i}^{\mathrm{e}}(j)=\frac{1}{h_{i}(j)} & \left(\sum_{\ell=1}^{\left\lfloor h_{i}(j)\right\rfloor} R_{i}^{\mathrm{e}}(j-\ell)\right. \\
& +R_{i}^{\mathrm{e}}\left(j-\left\lceil h_{i}(j)\right\rceil\right)\left(h_{i}(j)-\left\lfloor h_{i}(j)\right\rfloor\right)
\end{aligned}
$$

is the average encoding rate of the buffered VUs in buffer $i$ at time $j$ and $\lfloor\cdot\rfloor$ and $\lceil\cdot\rceil$ denote rounding towards $-\infty$ and $+\infty$. Since (6) still requires the availability of $h_{i}(j)$, we choose to estimate it as follows

$$
\begin{gathered}
\tilde{R}_{i}^{\mathrm{e}}(1)=R_{i}^{\mathrm{e}}(1) \\
\tilde{R}_{i}^{\mathrm{e}}(j+1)=\alpha R_{i}^{\mathrm{e}}(j)+(1-\alpha) \tilde{R}_{i}^{\mathrm{e}}(j), j>1
\end{gathered}
$$

where $0<\alpha<1$ is some tuning parameter. Then, one gets an estimate of the buffering delay $\tau_{i}(j)$ using (7)

$$
\tilde{\tau}_{i}(j)=B_{i}(j) / \tilde{R}_{i}^{\mathrm{e}}(j)
$$

\section{B. Rate controllers}

A centralized transmission rate controller and $N$ possibly decentralized encoding rate controllers are considered in the video delivery system described in Section II. PI controllers are used to regulate the transmission rate. At time $j$, these controllers take as input all utilities $U_{i}(j-1)$ of the previously encoded VUs, $i=1, \ldots, N$, that have reached the buffers in the MANE.

PI controllers are also used to evaluate the encoding rate target of each program in order to regulate the buffering delay around $\tau_{0}$. Each of these controllers takes as input the estimated buffering delay $\tilde{\tau}_{i}(j)$ provided by $(8)$ and the reference buffering delay $\tau_{0}$.

All rates are evaluated with respect to $R_{0}=R^{\mathrm{c}} / N$, the average encoding and transmission rate per stream, used if the $N$ streams represent the same encoded video.

1) Transmission rate control: At time $j$, the available transmission rate $R^{\mathrm{c}}$ is shared between video programs. The delayed utility information available at the MANE at time $j$ for the $i$-th program is

$$
U_{i}^{\mathrm{d}}(j)=U_{i}(j-1)
$$

and the discrepancy $\delta U_{i}^{\mathrm{d}}(j)$ with the average utility is

$$
\delta U_{i}^{\mathrm{d}}(j)=\frac{1}{N} \sum_{\ell=1}^{N}\left(U_{\ell}^{\mathrm{d}}(j)-U_{i}^{\mathrm{d}}(j)\right) .
$$

The PI transmission rate controller for the $i$-th program takes $\delta U_{i}^{\mathrm{d}}(j)$ to evaluate

$$
R_{i}^{\mathrm{t}}(j)=R_{0}+\left(K_{P}^{\mathrm{t}}+K_{I}^{\mathrm{t}}\right) \delta U_{i}^{\mathrm{d}}(j)+K_{I}^{\mathrm{t}} \phi_{i}(j),
$$

which is the $i$-th MANE buffer draining rate. In $(11), \phi_{i}(j)$ is the cumulated utility discrepancy (used for the integral term) evaluated as

$$
\phi_{i}(j+1)=\phi_{i}(j)+\delta U_{i}^{\mathrm{d}}(j), \text { with } \phi_{i}(0)=0 .
$$

$K_{\mathrm{P}}^{\mathrm{t}}$ and $K_{\mathrm{I}}^{\mathrm{t}}$ are the proportional and integral correction gains. One may easily prove that

$$
\sum_{i=1}^{N} R_{i}^{\mathrm{t}}(j)=R^{c}
$$

According to (11), in a first approximation, more (less) rate is allocated to programs with a utility below (above) average. 
2) Encoding rate control: At time $j$, the discrepancy $\delta \tilde{\tau}_{i}(j)$ between $\tilde{\tau}_{i}(j)$ and $\tau_{0}$ is

$$
\delta \tilde{\tau}_{i}(j)=\tilde{\tau}_{i}(j)-\tau_{0}=\left(\frac{B_{i}(j)}{\tilde{R}_{i}^{e}(j)}-\tau_{0}\right) .
$$

Buffers with positive $\delta \tau_{i}(j)$ are filling faster than draining. The encoding/transcoding rate $R_{i}^{\mathrm{e}}(j)$ has thus to be decreased to limit the increase of the buffering delay. This part of the control process is very similar to back-pressure algorithms [24].

Here, we choose again to evaluate $R_{i}^{\mathrm{e}}(j)$ as the output of a PI controller fed by $\delta \tilde{\tau}_{i}(j)$

$$
R_{i}^{\mathrm{e}}(j)=R_{0}-\frac{K_{P}^{\mathrm{e}}+K_{I}^{\mathrm{e}}}{T} \delta \tilde{\tau}_{i}(j)-\frac{K_{I}^{e}}{T} \Pi_{i}(j)
$$

where $\Pi_{i}(j)$ is the cumulated buffer delay discrepancy (used again for the integral term) evaluated as

$$
\Pi_{i}(j+1)=\Pi_{i}(j)+\delta \tilde{\tau}_{i}(j), \text { with } \Pi_{i}(0)=0 .
$$

$K_{\mathrm{P}}^{\mathrm{e}}$ and $K_{\mathrm{I}}^{\mathrm{e}}$ are the proportional and integral correction gains. According to (15), in a first approximation, the encoding rate increases (decreases) when the buffer is below (above) the reference delay $\tau_{0}$.

To account for the delayed utility available at the MANE, a state variable representing the delayed encoding rate is introduced

$$
R_{i}^{\mathrm{ed}}(j)=R_{i}^{\mathrm{e}}(j-1) .
$$

The sum of the encoding rates is not necessarily equal to $R^{\mathrm{c}}$. This allows to compensate for the variations of the video characteristics.

Combining (5), (11), and (15), one sees that buffers corresponding to programs producing video with lower utility than the average utility are drained faster. As a consequence, the encoding rate allowed to encode the next VU of such programs is increased.

\section{State-space representation}

To describe the evolution with time of the video delivery system, one has to take into account (1), (2), (7), (11), (12), (15), (16), and (17). One gets the following discrete-time nonlinear state-space representation

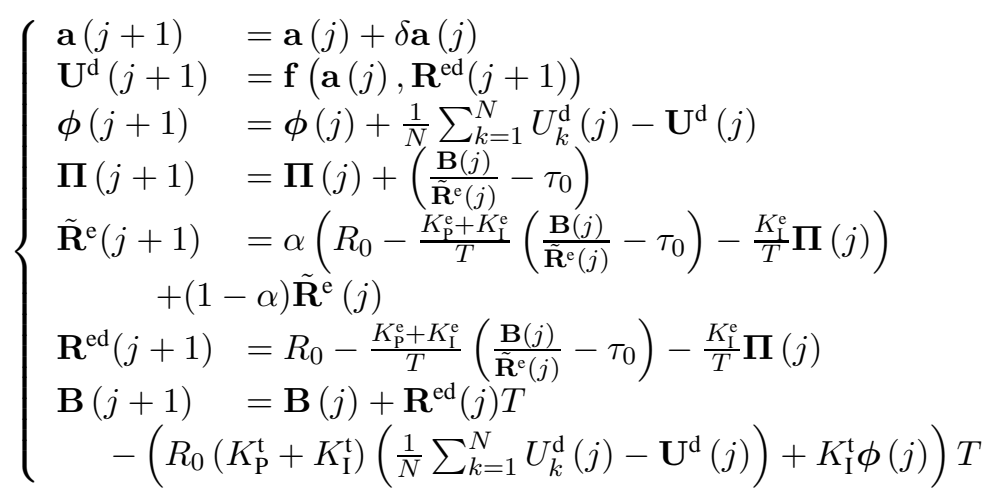

where all boldface letters are vectors, for example $\mathbf{B}(j)=\left(B_{1}(j) \ldots B_{N}(j)\right)^{\mathrm{T}}$ and $\mathbf{a}(j)=\left(\mathbf{a}_{1}^{\mathrm{T}}(j) \ldots \mathbf{a}_{N}^{\mathrm{T}}(j)\right)^{\mathrm{T}}$. In (18), the division $\mathbf{B}(j) / \tilde{\mathbf{R}}^{\mathrm{e}}(j)$ is component by component, as well as the difference between a scalar and a vector.

\section{EQUILIBRIUM AND STABILITY}

The steady-state behavior of the video delivery system described by (18) is studied first. Due to the coupling between controllers induced by the constraint that the discrepancy between the average utility and the utility of each program has to be as small as possible, the stability of the whole system has to be studied. This is performed by linearising (18) around its equilibrium. 


\section{A. Equilibrium analysis}

The system described by (18) reaches an equilibrium when all terms on the left of (18) do not change with time. This leads to the following system of $\left(6+N_{a}\right) N$ equations with $\left(6+N_{a}\right) N$ unknowns (a $\mathbf{a}^{\text {eq }}$ is assumed to be properly estimated in steady state)

$$
\begin{cases}\delta \mathbf{a}^{\mathrm{eq}} & =\mathbf{0} \\ \mathbf{U}^{\mathrm{eq}} & =\mathbf{f}\left(\mathbf{a}^{\mathrm{eq}}, \mathbf{R}^{\mathrm{eq}}\right) \\ \mathbf{U}^{\mathrm{eq}}-\frac{1}{N} \sum_{k=1}^{N} U_{k}^{\mathrm{eq}} & =\mathbf{0} \\ \mathbf{B}^{\mathrm{eq}} & =\tau_{0} \tilde{\mathbf{R}}^{\mathrm{eq}} \\ \tilde{\mathbf{R}}^{\mathrm{e} \text { eq }} & =R_{0}-\frac{K_{\mathrm{I}}^{\mathrm{e}}}{T^{\mathrm{eq}}} \\ \mathbf{R}^{\mathrm{eq}} & =R_{0}-\frac{K_{\mathrm{I}}^{\mathrm{eq}}}{T} \boldsymbol{\Pi}^{\mathrm{eq}} \\ \mathbf{R}^{\mathrm{eq}} & =K_{\mathrm{I}}^{\mathrm{t}} \phi^{\mathrm{eq}}\end{cases}
$$

The third equation in (19) imposes that $U_{1}^{\mathrm{eq}}=\cdots=U_{N}^{\mathrm{eq}}=U^{\mathrm{eq}}$. All programs have thus the same utility at equilibrium. One has $\tilde{\mathbf{R}}^{\mathrm{e} \text { eq }}=\mathbf{R}^{\mathrm{eq}}$ and $\mathbf{B}^{\mathrm{eq}}=\tau_{0} \mathbf{R}^{\mathrm{eq}}$, which leads to $B_{i}^{\mathrm{eq}} / R_{i}^{\mathrm{eq}}=\tau_{i}(j)=\tau_{0}, i=1, \ldots, N$ meaning that at equilibrium, the buffering delay is equal to $\tau_{0}$ for all streams.

Since $f$ is strictly increasing in $R$, the rate at equilibrium as a function of $U^{\text {eq }}$ is

$$
R_{i}^{\mathrm{eq}}=f_{R}^{-1}\left(\mathbf{a}^{\mathrm{eq}}, U^{\mathrm{eq}}\right), i=1, \ldots, N
$$

with $f_{R}^{-1}$ is the inverse of $f$ seen as a function of $R$ only. The value of $U^{\text {eq }}$ is determined from the channel rate constraint

$$
\sum_{i=1}^{N} R_{i}^{\mathrm{eq}}=\sum_{i=1}^{N} f_{R}^{-1}\left(\mathbf{a}_{i}^{\mathrm{eq}}, U^{\mathrm{eq}}\right)=R^{c}
$$

Since $f(\mathbf{a}, R)$ is continuous and strictly increasing in $R, f_{R}^{-1}(\mathbf{a}, U)$ and $\sum_{i=1}^{N} f_{R}^{-1}\left(\mathbf{a}_{i}, U\right)$ are also continuous and strictly increasing functions of $U$, with $\sum_{i=1}^{N} f_{R}^{-1}\left(\mathbf{a}_{i}, 0\right)=0$. Provided that $\lim _{U \rightarrow \infty} \sum_{i=1}^{N} f_{R}^{-1}\left(\mathbf{a}_{i}, U\right)>R^{c},(21)$ admits a single solution. Then, $\Pi^{\mathrm{eq}}$ and $\phi^{\mathrm{eq}}$ are deduced from (19) and (20), provided that $K_{\mathrm{I}}^{\mathrm{e}} \neq 0$ and $K_{\mathrm{I}}^{\mathrm{t}} \neq 0$.

The equilibrium is thus unique and the two control loops lead to a satisfaction of the targets introduced in Section II.

\section{B. Stability}

The stability analysis of (18) is performed considering its linearization around the equilibrium obtained from (19)

$$
\left\{\begin{array}{c}
\Delta \mathbf{a}(j+1)=\Delta \mathbf{a}(j)+\delta \mathbf{a}(j) \\
\Delta \boldsymbol{\phi}(j+1)=\Delta \boldsymbol{\phi}(j)+\frac{1}{N} \sum_{k=1}^{N} \Delta U_{k}(j)-\Delta \mathbf{U}^{\mathrm{d}}(j) \\
\Delta \mathbf{\Pi}(j+1)=\Delta \boldsymbol{\Pi}(j)-\mathbf{V}\left(\tau_{0} \Delta \tilde{\mathbf{R}}^{\mathrm{e}}(j)-\Delta \mathbf{B}(j)\right) \\
\Delta \tilde{\mathbf{R}}^{\mathrm{e}}(j+1)=(1-\alpha) \Delta \tilde{\mathbf{R}}^{\mathrm{e}}(j) \\
\quad+\alpha\left(\frac{K_{\mathbf{P}}^{\mathrm{e}}+K_{\mathrm{I}}^{\mathrm{e}}}{T} \mathbf{V}\left(\tau_{0} \Delta \tilde{\mathbf{R}}^{\mathrm{e}}(j)-\Delta \mathbf{B}(j)\right)-\frac{K_{\mathrm{I}}^{\mathrm{e}}}{T} \Delta \mathbf{\Pi}(j)\right) \\
\Delta \mathbf{R}^{\mathrm{ed}}(j+1)=\frac{K_{\mathrm{P}}^{\mathrm{e}}+K_{\mathrm{I}}^{\mathrm{e}}}{T} \mathbf{V}\left(\tau_{0} \Delta \tilde{\mathbf{R}}^{\mathrm{e}}(j)-\Delta \mathbf{B}(j)\right)-\frac{K_{\mathrm{I}}^{\mathrm{e}}}{T} \Delta \mathbf{\Pi}(j) \\
\Delta \mathbf{U}^{\mathrm{d}}(j+1)=\frac{\partial \mathbf{f}}{\partial \mathbf{a}^{T}}\left(\mathbf{a}^{\mathrm{eq}}, \mathbf{R}^{\mathrm{eq}}\right) \Delta \mathbf{a}(j) \\
\quad+\frac{\partial \mathbf{f}}{\partial \mathbf{R}^{T}}\left(\mathbf{a}^{\mathrm{eq}}, \mathbf{R}^{\mathrm{eq}}\right)\left(\frac{K_{\mathbf{P}}^{\mathrm{e}}+K_{\mathrm{I}}^{\mathrm{e}}}{T} \mathbf{V}\left(\tau_{0} \Delta \tilde{\mathbf{R}}^{\mathrm{e}}(j)-\Delta \mathbf{B}(j)\right)-\frac{K_{\mathrm{I}}^{\mathrm{e}}}{T} \Delta \mathbf{\Pi}(j)\right) \\
\Delta \mathbf{B}(j+1)=\Delta \mathbf{B}(j)+\Delta \mathbf{R}^{\mathrm{ed}}(j) T \\
\quad-\left(\left(K_{\mathrm{P}}^{\mathrm{t}}+K_{\mathrm{I}}^{\mathrm{t}}\right)\left(\frac{1}{N} \sum_{k=1}^{N} \Delta U_{k}^{\mathrm{d}}(j)-\Delta \mathbf{U}^{\mathrm{d}}(j)\right)+K_{\mathrm{I}}^{\mathrm{t}} \Delta \phi(j)\right) T
\end{array}\right.
$$

where $\mathbf{V}=\operatorname{diag}\left(1 / R_{1}^{\mathrm{eq}}, \ldots, 1 / R_{N}^{\mathrm{eq}}\right)$ is a diagonal matrix containing the inverse of the encoding rates at equilibrium. The state vector of the linearized system is

$$
\begin{aligned}
\mathbf{x}(j)= & (\Delta \mathbf{a}(j), \Delta \boldsymbol{\phi}(j), \Delta \mathbf{\Pi}(j), \\
& \left.\Delta \tilde{\mathbf{R}}^{\mathrm{e}}(j), \Delta \mathbf{R}^{\mathrm{ed}}(j), \Delta \mathbf{U}^{\mathrm{d}}(j), \Delta \mathbf{B}(j)\right)^{T} .
\end{aligned}
$$


Introduce the $N \times\left(N \times N^{a}\right)$ block diagonal matrix

$$
\begin{aligned}
\Xi & =\frac{\partial \mathbf{f}}{\partial \mathbf{a}^{T}}\left(\mathbf{a}^{\mathrm{eq}}, \mathbf{R}^{\mathrm{eq}}\right) \\
& =\operatorname{diag}\left(\frac{\partial f}{\partial \mathbf{a}_{1}^{T}}\left(\mathbf{a}_{1}^{\mathrm{eq}}, R_{1}^{\mathrm{eq}}\right), \ldots, \frac{\partial f}{\partial \mathbf{a}_{N}^{T}}\left(\mathbf{a}_{N}^{\mathrm{eq}}, R_{N}^{\mathrm{eq}}\right)\right),
\end{aligned}
$$

the $N \times N$ diagonal matrix

$$
\begin{aligned}
\boldsymbol{\Gamma} & =\frac{\partial \mathbf{f}}{\partial \mathbf{R}^{T}}\left(\mathbf{a}^{\mathrm{eq}}, \mathbf{R}^{\mathrm{eq}}\right) \\
& =\operatorname{diag}\left(\frac{\partial f}{\partial R_{1}}\left(\mathbf{a}_{1}^{\mathrm{eq}}, R_{1}^{\mathrm{eq}}\right), \ldots, \frac{\partial f}{\partial R_{N}}\left(\mathbf{a}_{N}^{\mathrm{eq}}, R_{N}^{\mathrm{eq}}\right)\right),
\end{aligned}
$$

and the $N \times N$ circulant matrix

$$
\mathbf{L}=\frac{1}{N} \operatorname{toeplitz}(N-1,-1, \ldots,-1) .
$$

$\mathbf{I}$ and $\mathbf{0}$ are the identity and null matrix of appropriate size. One then gets from (22) the linear discrete-time state equation

$$
\mathbf{x}(j+1)=\mathbf{A} \mathbf{x}(j)+\mathbf{w}(j)
$$

where

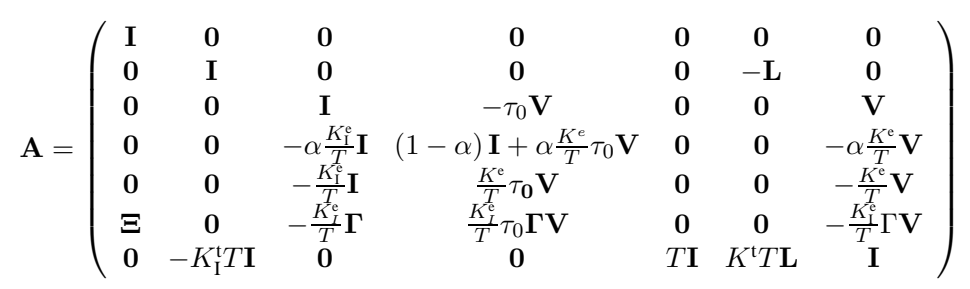

with $K^{\mathrm{e}}=K_{\mathrm{P}}^{\mathrm{e}}+K_{\mathrm{I}}^{\mathrm{e}}, K^{\mathrm{t}}=K_{\mathrm{P}}^{\mathrm{t}}+K_{\mathrm{I}}^{\mathrm{t}}$, and the perturbation

$$
\mathbf{w}(j)=(\delta \mathbf{a}(j), 0, \ldots 0)^{T} .
$$

The stability of the system described by (18) is determined by the location of the roots of $\operatorname{det}(z \mathbf{I}-\mathbf{A})$ with respect to the unit circle. After some derivations, one obtains

$$
\operatorname{det}(z \mathbf{I}-\mathbf{A})=\left(\prod_{i=1}^{N} \frac{1}{R_{i}^{\mathrm{eq}}}\right) z^{N}(z-1)^{N\left(2+N_{\mathrm{a}}\right)} \operatorname{det} \mathbf{F},
$$

with

$$
\begin{aligned}
\mathbf{F}= & -P_{6}(z) P_{3}(z)\left(P_{1}(z) \mathbf{V}^{-1}-\alpha \tau_{0} P_{2}(z) \mathbf{I}\right) \\
& P_{1}(z) P_{2}(z) P_{4}(z) \mathbf{I}+P_{1}(z) P_{2}(z) P_{5}(z) \Gamma \mathbf{L}
\end{aligned}
$$

and

$$
\begin{array}{ll}
P_{1}(z)=(z-1)(z-1+\alpha) & P_{2}(z)=(z-1) \frac{K^{e}}{T}+\frac{K_{\mathrm{I}}^{\mathrm{e}}}{T} \\
P_{3}(z)=z(z-1) & P_{4}(z)=-(z-1) T, \\
P_{5}(z)=-(z-1) K^{\mathrm{t}} T-K_{\mathrm{I}}^{\mathrm{t}} T & P_{6}(z)=(z-1)^{2} .
\end{array}
$$

depend on the parameters of the two PI controllers. The $N\left(2+N_{\mathrm{a}}\right)$ zeros at $z=1$ of (28) correspond to the evolution of the source parameters and to the integral terms. The evaluation of $\operatorname{det} \mathbf{F}$ in (28) may only be done numerically. However, (28) may be simplified assuming that all programs have the same characteristics, which leads to $\Gamma=\gamma \mathbf{I}$ and $\mathbf{V}=\frac{1}{R_{0}} \mathbf{I}$. In this case, $\mathbf{F}=D(z) \mathbf{I}+K(z) \mathbf{L}$ with

$$
D(z)=P_{1}(z) P_{2}(z) P_{4}(z)-P_{6}(z) P_{3}(z)\left(R_{0} P_{1}(z)-\alpha \tau_{0} P_{2}(z)\right)
$$

and

$$
K(z)=\gamma P_{1}(z) P_{2}(z) P_{5}(z)
$$




\begin{tabular}{|c|c|c|c|}
\hline Video & Rate(kbit/s) & PSNR (dB) & Activity \\
\hline Prog 1 & 1669.9 & 46.06 & low \\
\hline Prog 2 & 4929.1 & 44.23 & high \\
\hline Prog 3 & 3654.6 & 44.56 & medium \\
\hline Prog 4 & 2915.1 & 44.61 & low \\
\hline
\end{tabular}

TABLE I

AVERAGE RATE AND CORRESPONDING PSNR OF THE FOUR CONSIDERED VIDEO STREAMS

$$
\begin{aligned}
\operatorname{det} \mathbf{F} & =\operatorname{det}\left(D(z) \mathbf{I}+\frac{1}{N} K(z)\left(\begin{array}{ccc}
N-1 & -1 & -1 \\
-1 & \ddots & -1 \\
-1 & -1 & N-1
\end{array}\right)\right) \\
& =(K(z)+D(z))^{N}
\end{aligned}
$$

One has thus to study the location of the zeros of $K(z)+D(z)$, which allow to predict whether a given choice of $K_{\mathrm{P}}^{\mathrm{t}}, K_{\mathrm{I}}^{\mathrm{t}}, K_{\mathrm{P}}^{\mathrm{e}}$, and $K_{\mathrm{I}}^{\mathrm{e}}$ leads to a stable or to an unstable system, and help to predict the transient evolution of the system.

\section{ApPlicAtion CONTEXT}

A typical application scenario for the proposed rate control system is the Mobile TV service delivery using the evolved MBMS standard [25]. Here, we briefly describe the functional architecture of the multiplexing functions. Detailed implementation issues are not addressed.

MBMS is a point-to-multipoint interface specification for 3GPP cellular networks, which is designed to provide efficient delivery of broadcast and multicast services. For broadcast transmission, a single frequency network configuration is introduced in 3GPP LTE specifications which enables a time synchronization between a set of eNBs (base stations) using the same resource block.

The MBMS architecture is composed of three main entities: BM-SC, MBMS-GW and MCE. The Multicast/Broadcast Service Center (BM-SC) is a node that serves as an entry point for the content providers delivering the video sources, used for service announcements, session management. The MANE, considered in the paper in charge of choosing the encoding and the transmission rates, may be located in the BM-SC node. The MBMS-Gateway (GW) is an entity responsible for distributing the traffic across the different eNBs belonging to the same broadcast area. It ensures that the same content is sent from all the eNBs by using IP Multicast. The Multi-cell/multicast Coordination Entity (MCE) is a logical entity, responsible for allocation of time and frequency resources for multicell MBMS transmission. As in [26], we assume that the MBMS-GW periodically notifies the MCE about the resource requirements of video streams so that the resources at eNBs can be re-allocated accordingly. Therefore, the BM-SC should ensure that the encoding rate of the multiplex does not violate the already allocated resources. This is obtained thanks to the proposed rate control scheme.

\section{Simulation RESULTS}

This section considers a typical application context presented in Section V concerning mobile TV service delivery over evolved MBMS standard. $N=4$ video streams, each of $100 \mathrm{~s}$ long, extracted from real TV programs: News (Prog 1), Sport (Prog 2), Big Buck Bunny (Prog 3), and Nature Documentary (Prog 4) in 4CIF (704 $\times 576)$ format are encoded with x.264 [27] at a frame rate of $F=30$ frames/s. GoPs of 15 frames are considered, thus the GoP duration is $T=0.5 \mathrm{~s}$. The videos are already encoded using MPEG-4 and then they have been converted to YUV format using ffmpeg [28]. The four videos are then processed with the proposed control system. The control is performed at the GoP level. A summary of the video characteristics is provided in Table I.

Initially, all buffers contain two encoded GoPs corresponding to a buffer delay of $1 \mathrm{~s}$. The reference delay is taken as $\tau_{0}=2 \mathrm{~s}$. The encoding/transcoding rate (15) is sent to the video encoders which have to choose the encoding parameters for the VU. In this simulation part, the video quality is measured using the PSNR or the SSIM associated to the encoded VUs. This quality metric is transmitted to the MANE in the packet headers. Note that the utility model (1) is only required to characterize the stability of the system and to tune the control parameters. Once the parameters have been chosen, there is no need to know precisely the model (1). 

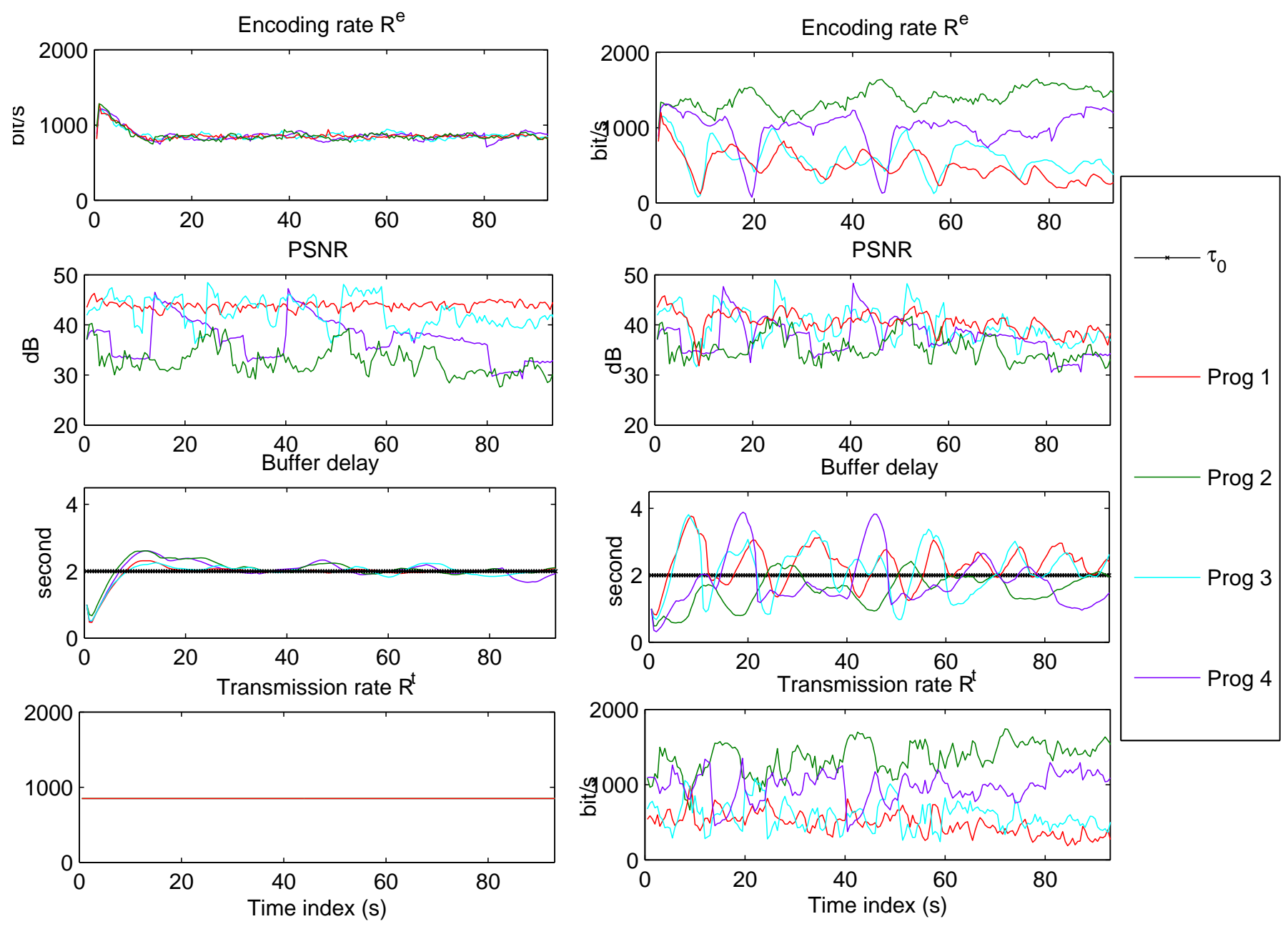

Fig. 3. System performance using PI controllers while multiplexing four video programs using the proposed QF solution (left) and the TRF system (right).

\section{A. Constant channel rate and number of users}

Here, the four video programs are encoded and transmitted: News (Prog 1), Sport (Prog 2), Cartoon (Prog 3), and Documentary (Prog 4). A constant channel rate $R^{\mathrm{c}}=3.5 \mathrm{Mbit} / \mathrm{s}$ is considered. The maximum buffer size is $B^{\max }=4$ Mbits. The proposed quality fair (QF) video delivery system is compared to a classical scheme which provide equal transmission rate to the $N$ video streams. This scheme controls the encoding rate as in (15) to limit the buffering delay, this solution is called transmission rate fair (TRF) video delivery system.

In order to choose the parameters of all PI controllers and to characterize the stability region, as in[29], [30] a linear PSNR-Rate model is considered so that

$$
P_{i}(j)=U_{i}(j)=f\left(\mathbf{a}_{i}(j), R_{i}^{\mathrm{e}}(j)\right)=a_{i}^{1}(j) R_{i}^{\mathrm{e}}(j)+a_{i}^{2}(j)
$$

where $P_{i}(j)$ is the PSNR of program $i$ at time $j$ and the element of the vector $\mathbf{a}_{i}(j)$ are derived using some encoding trials for the $N$ considered programs. Using $f$, one can derive the system equilibrium and the stability of the control system. Parameters of the two PI controllers are set in such a way that the roots of $\operatorname{det}(z \mathbf{I}-\mathbf{A})=0$ are within the unit circle. Among the values of the parameters leading to a stable system, one may choose the values that provides good performance in terms of both buffering delay and PSNR discrepancy and variance. Good transient behaviors have been obtained with $K_{\mathrm{P}}^{\mathrm{t}}=4250, K_{\mathrm{I}}^{\mathrm{t}}=425 K_{\mathrm{P}}^{\mathrm{e}}=127500$, and $K_{\mathrm{I}}^{\mathrm{e}}=3400$.

The PSNRs $P_{i}(j)$, the buffering delays $\tau_{i}(j)$, the encoding rates $R_{i}^{\mathrm{e}}(j)$, and the transmission rates $R_{i}^{\mathrm{t}}(j)$ of each video stream are represented in Figure 3 (left) using the proposed QF solution and in Figure 3 (right) using the TRF solution when quality fairness is performed in term of PSNR. 
The tuning parameter $\alpha$ used to estimate the buffering delay has been set to $\alpha=0.7$ corresponding to the least mean squared error between the real buffer delay $\tau$ and the estimated buffer delay $\tilde{\tau}$. Table II represents $M S E(\tilde{\tau}, \tau)$ as a function of $\alpha$. The $\mathrm{x} 264$ video encoder is used to encode on-line the different programs according to the target

\begin{tabular}{|c|c|c|c|}
\hline$\alpha$ & $M S E(\tilde{\tau}, \tau)$ & $\alpha$ & $M S E(\tilde{\tau}, \tau)$ \\
\hline 0.5 & 0.0035 & 0.6 & 0.0021 \\
\hline 0.65 & 0.0016 & 0.7 & 0.0014 \\
\hline 0.75 & 0.0019 & 0.8 & 0.0035 \\
\hline
\end{tabular}

TABLE II

MSE OF THE ESTIMATED BUFFERING DELAY AS A FUNCTION OF $\alpha$.

rate constraint derived by the individual encoding rate controllers. Thus, the rate control function in each video encoder is activated. A two pass control process in the rate control is performed to better fit the target bit rate and reduce the rate discrepancies. However, the obtained rate may be slightly different from that required by the MANE which may introduce some oscillations.

The system performance is measured in terms of average discrepancy $\Delta_{\tau}$ (in s) of the buffering delay with respect to $\tau_{0}$, variance of the buffer delay $\sigma_{\tau}^{2}$ (in $\mathrm{s}^{2}$ ), PSNR discrepancy $\Delta_{P}$ (in $\mathrm{dB}$ ), and PSNR variance $\sigma_{P}^{2}$ (in $\mathrm{dB}^{2}$ ), defined by

$$
\begin{array}{r}
\Delta_{\tau}=\mid \frac{1}{N} \sum_{n=1}^{N}\left(\frac{1}{M} \sum_{l=1}^{M}\left(\tau_{n}(l)-\tau_{0}\right)\right), \\
\sigma_{\tau}^{2}=\frac{1}{N} \sum_{n=1}^{N}\left(\frac{1}{M} \sum_{l=1}^{M}\left(\tau_{n}(l)-\tau_{0}\right)^{2}\right), \\
\sigma_{P S N R}^{2}=\frac{1}{N} \sum_{n=1}^{N}\left(\frac{1}{M} \sum_{l=1}^{M}\left(P_{n}(l)-\bar{P}(l)\right)^{2}\right), \\
\Delta_{P S N R}=\mid \frac{1}{N} \sum_{n=1}^{N}\left(\frac{1}{M} \sum_{l=1}^{M}\left(P_{n}(l)-\bar{P}(l)\right)\right),
\end{array}
$$

where $M$ is the number of GoPs in the video streams.

In Figure 3, one can see that the proposed QF solution provides a significant reduction of the PSNR discrepancy between the four programs compared to the TRF solution. The QF solution reduces the mean PSNR discrepancy to $\Delta_{P S N R}=2.2 \mathrm{~dB}$ compared to $\Delta_{P S N R}=4.15 \mathrm{~dB}$ using the TRF solution.

Results are summarized in Table III in three cases: (i) Only $\mathrm{P}$ controllers are used (P, P), (ii) P controller is used for the transmission rate control and PI controller for the encoding rate control (P, PI), and (iii) PI controllers are used (PI, PI). The PI controller in the encoder rate control loops significantly decreases the discrepancy of the buffering delay $\Delta_{\tau}$ compared to a situation where only $\mathrm{P}$ controllers are used, without disturbing the behavior of the PSNR regulation. In addition, using PI controllers for the transmission rate control loop reduces the PSNR discrepancy between the programs at the price of some increase of the buffer delay discrepancy and variance.

\begin{tabular}{|c|c|c|c|c|c|}
\hline$K_{\mathrm{P}}^{\mathrm{l}}, K_{\mathrm{I}}^{\mathrm{l}}$ & $K_{\mathrm{P}}^{\mathrm{e}}, K_{\mathrm{I}}^{\mathrm{e}}$ & $\Delta_{\tau}$ & $\sigma_{\tau}^{2}$ & $\Delta_{P}$ & $\sigma_{P}^{2}$ \\
\hline 4250,0 & 127500,0 & 0.1 & 0.5 & 4.15 & 11.2 \\
\hline 4250,0 & 127500,3400 & 0.008 & 0.19 & 4 & 10.9 \\
\hline 4250,425 & 127500,3400 & 0.01 & 0.22 & 2.2 & 11.18 \\
\hline
\end{tabular}

TABLE III

Performance When using (P, P), (P, PI), AND (PI, PI) CONTROLlERS IN THE PRoposed QF VIDEO DELIVERY SYSTEM.

One can also notice that the average PSNR of Prog 2, characterized by high activity level, is improved from $32 \mathrm{~dB}$ to $37 \mathrm{~dB}$ which corresponds to a significant improvement of the subjective video quality. This improvement is at the expense of PSNR degradation of Prog 1, characterized by low activity level. The average PSNR of Prog 1 decreases from $47 \mathrm{~dB}$ to $42 \mathrm{~dB}$ which still correspond to a very good quality. On the other hand, the discrepancy 

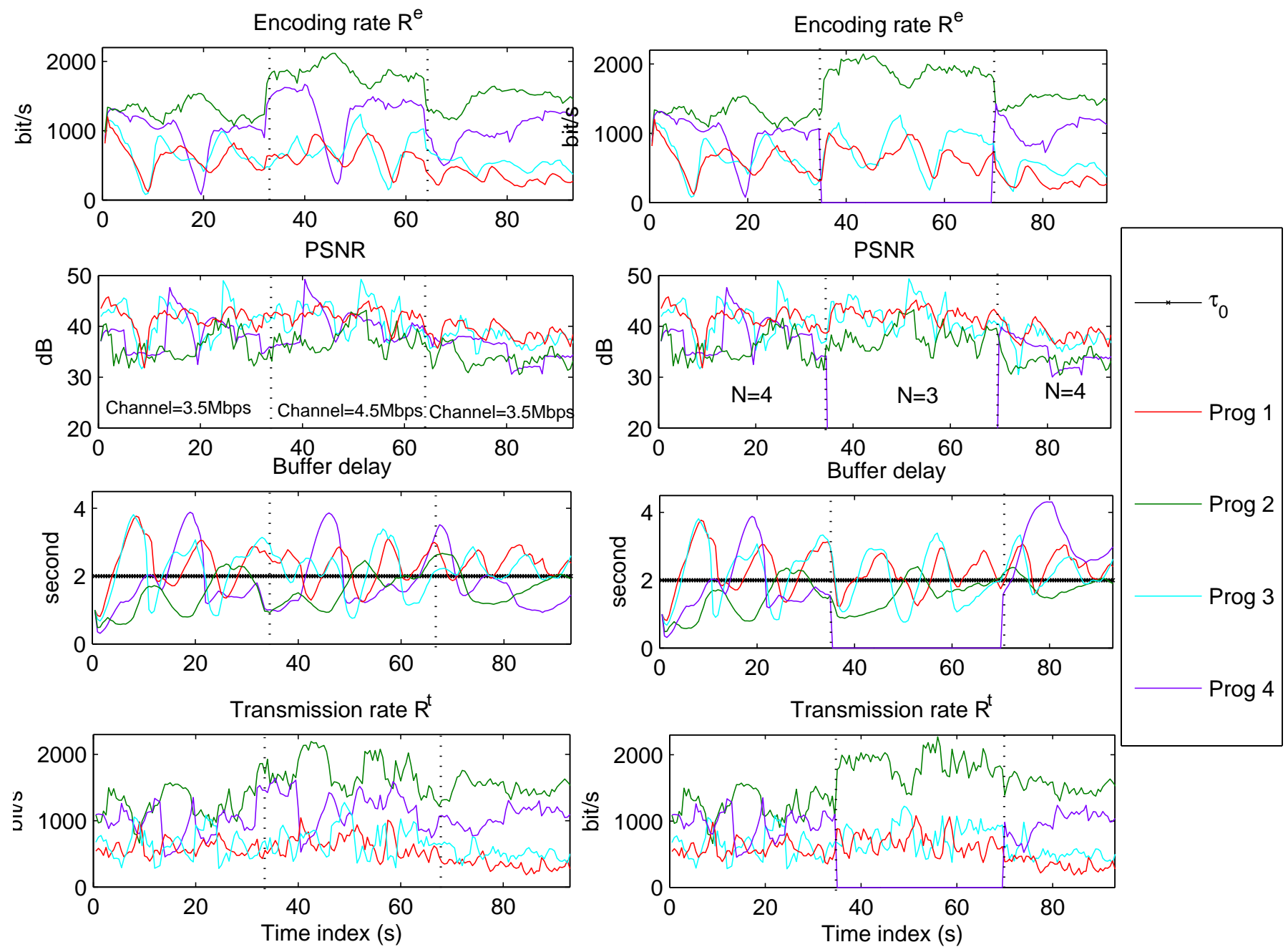

Fig. 4. System performance using PI controllers while multiplexing four video programs using the proposed QF solution when considering variations of the channel rate (left) and of the number of programs (right).

between the buffering delay and $\tau_{0}$ remains limited for most of the time. The buffering delay variations are due to the interactions of both control loops (encoding rate) and (transmission rate) using two PI controllers. When only the encoding rate is controlled, corresponding to the TRF solution, the buffering delay reaches rapidly $\tau_{0}$.

\section{B. Robustness of the control system}

In this section, we analyze the robustness of the proposed control system while considering variations of the channel rate, of the number of video programs, as well as an other utility metric.

1) Variation of the channel rate and the number of video programs: First, the rate of the channel is varying between $R^{c}=3.5 \mathrm{Mbits} / \mathrm{s}$ and $R^{c}=4.5 \mathrm{Mbits} / \mathrm{s}$, see Figure 4 (left). Second, the number of multiplexed video programs is varying with time. This scenario corresponds to a change in the number of users. When a new video program is added to the multiplex, it has no bit rate allocated by the MANE to it (since at time $j$ the controller derives the encoding rate for time $j+1$ ). Thus, we choose to set the encoding rate at that time is $R^{\mathrm{c}} / N$. In Figure 4 (right), Prog 4 is not transmitted between GoP 35 and 65.

When the channel rate increases or when a video program is no more transmitted, the bandwidth allocation adapts rapidly to this change by allocating more rate to programs with low video quality (here Prog 2). When the channel rate decreases or when a new video program is added transmitted, the bandwidth allocation performs well, showing the robustness of the proposed control system to variations of the channel rate and the number of transmitted video programs. 
TRF constant channel
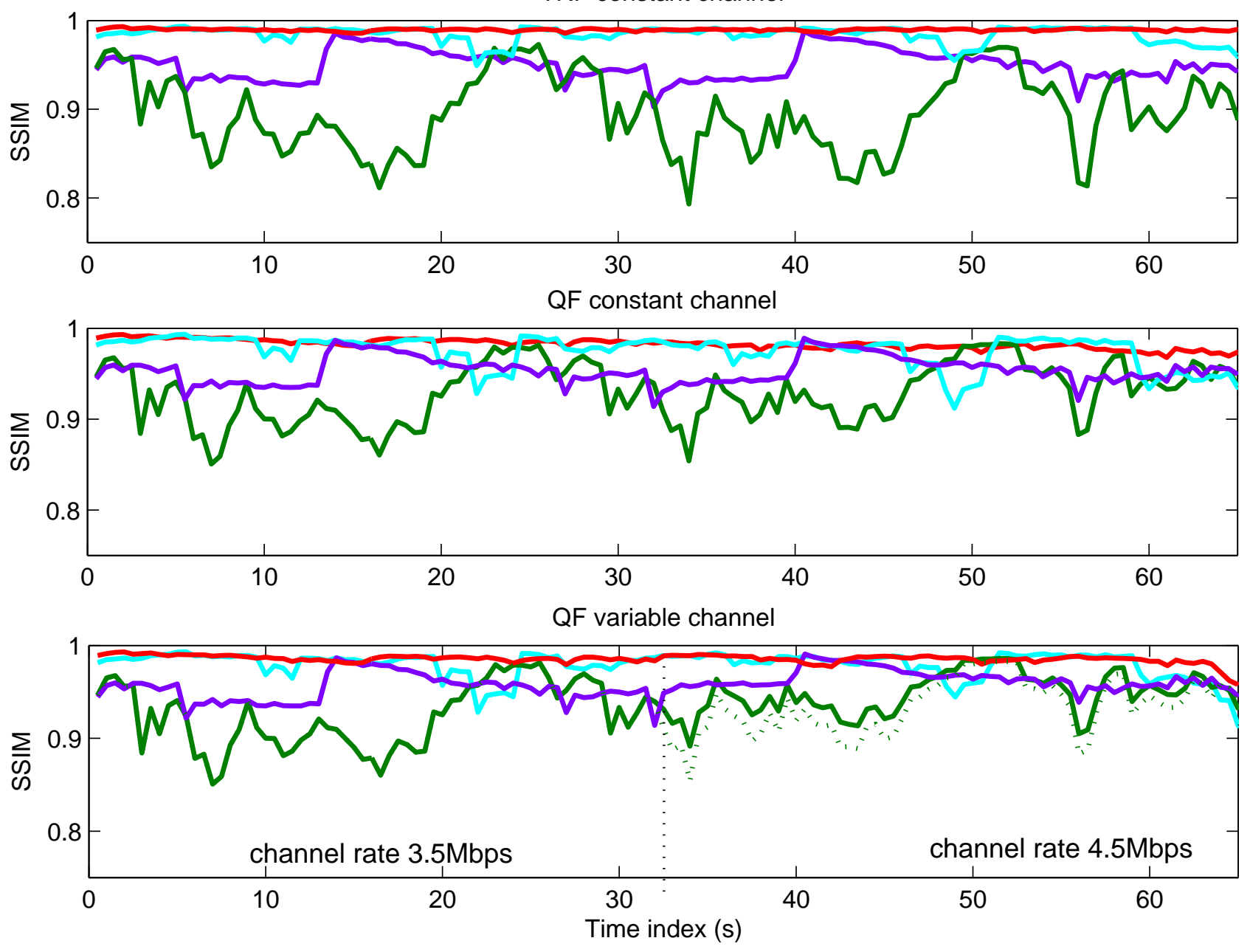

$\longrightarrow$ Prog $1 \longrightarrow$ Prog $2=$ Prog $3 \longrightarrow$ Prog 4

Fig. 5. System performance using PI controllers while multiplexing four video programs using the proposed QF solution when fairness in SSIM is considered for both constant and variable channel rates.

2) SSIM fairness: Here the quality fairness is addressed using the SSIM metric. In order to choose the parameters of all PI controllers and to characterize the stability region, a linear SSIM-Rate model is considered as in [31] so that

$$
S_{i}(j)=U_{i}(j)=f\left(\mathbf{a}_{i}(j), R_{i}^{\mathrm{e}}(j)\right)=a_{i}^{1}(j) R_{i}^{\mathrm{e}}(j)+a_{i}^{2}(j)
$$

where $S_{i}(j)$ is the SSIM of program $i$ at time $j$ and the element of the vector $\mathbf{a}_{i}(j)$ are derived using some encoding trials for the $N$ considered programs. A stable system and good transient behaviors have been obtained with $K_{\mathrm{P}}^{\mathrm{t}}=4250, K_{\mathrm{I}}^{\mathrm{t}}=425 K_{\mathrm{P}}^{\mathrm{e}}=127500$, and $K_{\mathrm{I}}^{\mathrm{e}}=3400$.

The SSIMs $S_{i}(j)$ of each program are represented in Figure 5 using the TRF, then using the proposed QF solution when the channel rate is constant and when it is time varying. Again the proposed QF solution reduces the SSIM discrepancy between the four programs compared to the TRF solution. Using the proposed solution the mean SSIM discrepancy is reduced to $\Delta_{S S I M}=0.02$ compared to $\Delta_{S S I M}=0.05$ with the TRF solution.

SSIM or PSNR are generated by the video encoder at each encoding process and transmitted to the MANE for the control process. Thus the control process does not use any trade off function between the rate and the video quality metric. Other test with subjective video quality fairness can be performed while using QoE-Rate model such as those in [32]. In this case, given the QoE-Rate trade off function, the equilibrium and the stability of the system can be characterized. 


\section{CONCLUSIONS AND PERSPECTIVES}

In this paper, we propose a control system for the encoding and the transmission of multiple video streams targeting similar video quality between streams as well as efficient buffering delay control. The control system is modeled with a discrete-time non-linear state-space representation. PI controllers for both the transmission rate control and the encoding rate control are considered. Simulation tests show that the quality fairness (measured with PSNR or SSIM) is improved compared to a solution providing a fair transmission rate allocation. Moreover, the jitter of the buffering delay remains reasonable.

This control system can be performed on scalable video sources. The same buffer and rate control system can be used to determine the optimal encoding rate for each video server. Only the rate control algorithm has to be adapted to fit the target bit rate. This can be done by adjusting the number of scalable layers. The main difficulty comes from the fact that not all rate points may be reached in a continuous way (this would require very fine grain scalability).

Control at the frame level should be considered, however this would require to much better model the packet delivery delays between the MANE and the encoders.

\section{ACKNOWLEDGMENTS}

This work was supported by ANR ARSSO project (grant agreement no 09-VERS-019). Michel Kieffer is partly supported by the Institut Universitaire de France

\section{REFERENCES}

[1] "Cisco visual networking index: Forecast and methodology, 2008-2013," Tech. Rep., Cisco, 2009.

[2] Z.L. Zhang, S. Nelakuditi, R. Aggarwal, and R.P. Tsang, "Efficient selective frame discard algorithms for stored video delivery across resource constrained networks," Real-Time Imaging, vol. 7, no. 3, pp. 255 - 273, 2001.

[3] T-L. Lin, Y. Zhi, S. Kanumuri, P.C. Cosman, and A.R. Reibman, "Perceptual quality based packet dropping for generalized video gop structures," in Proc. IEEE ICASSP, 2009, pp. 781 - 784.

[4] S. Ma, W. Gao, and Y. Lu, "Rate-distortion analysis for H.264/AVC video coding and its application to rate control," IEEE trans. on Circ. Syst. Vid. Techn., vol. 15, no. 12, pp. 1533 - 1544, 2005.

[5] Z. He and D. O. Wu, "Linear rate control and optimum statistical multiplexing for H.264 video broadcast," IEEE trans. on Multimedia, vol. 10, no. 7, pp. 1237 - 1249, 2008.

[6] S. K. Srinivasan, J. Vahabzadeh-Hagh, and M. Reisslein, "The effects of priority levels and buffering on the statistical multiplexing of single-layer H.264/AVC and SVC encoded video streams," IEEE trans. on Broadcasting, vol. 56, no. 3, pp. 281 - $287,2010$.

[7] E. Maani and A.K. Katsaggelos, "Unequal error protection for robust streaming of scalable video over packet lossy networks," IEEE trans. on Circ. Syst. Vid. Techn., vol. 20, no. 3, pp. 407 - 416, 2010.

[8] Y. Li, Z. Li, M. Chiang, and A.R. Calderbank, "Content-aware distortion-fair video streaming in congested networks," IEEE trans. on Multimedia, vol. 11, no. 6, pp. 1182 - 1193, 2009.

[9] N. Changuel, B. Sayadi, and M. Kieffer, "Joint encoder and buffer control for statistical multiplexing of multimedia contents," in Proc. IEEE Globecom, 2010, pp. 1 - 6.

[10] C.W Wong, O.C. Au, and H.K. Lam, "PID-based real-time rate control," in Proc. IEEE ICME, 2004, vol. 1, pp. 221 - 224.

[11] Z. Guo and R. Rojas-Cessa, "Analysis of a flow control system for a combined input-crosspoint buffered packet switch," in Workshop on High Performance Switching and Routing, 2005, pp. 336 - 340.

[12] Y-H. Zhang, K.H Li, C.Q. Xu, and L.M. Sun, "Joint rate allocation and buffer management for robust transmission of VBR video," Acta Automatica Sinica, vol. 34, no. 3, pp. 1 - 7, 2008.

[13] Y. Huang, S. Mao, and S.F. Midkiff, "A control-theoretic approach to rate control for streaming videos," IEEE trans. on Multimedia, vol. 11, no. 6, pp. 1072 - 1081, 2009.

[14] Y. Huang and S. Mao, "Analysis and design of a proportional-integral rate controller for streaming videos," in Proc. IEEE Globecom, 2009, pp. $5530-5535$.

[15] Y. Zhou, Y. Sun, Z. Feng, and S. Sun, "PID-based bit allocation strategy for h.264/avc rate control," IEEE trans. on Circ. Syst. Vid. Techn., vol. 58, no. 3, pp. $184-188,2011$.

[16] J.W Cho and S. Chong, "Utility max-min flow control using slope-restricted utility functions," in Proc. IEEE Globecom, 2005, vol. 2, pp. $818-824$.

[17] E. Witrant, C. Canudas-de Wit, D. Georges, and M. Alamir, "Remote stabilization via communication networks with a distributed control law," IEEE trans. on Autom. Contr., vol. 52, no. 8, pp. 1480 - 1485, 2007.

[18] Motorola, "Statistical multiplexing over IP StatmuxIP solution," Tech. Rep., Motorola, 2009, XP002565902.

[19] Harmonic, "IP-based distributed statistical multiplexing solution," Tech. Rep., 2011.

[20] K. Seshadrinathan, R. Soundararajan, A.C. Bovik, and L.K. Cormack, "Study of subjective and objective quality assessment of video," IEEE trans. on Image Proc., vol. 19, no. 6, pp. 1427 - 1441, 2010.

[21] T. Wiegand, H. Schwarz, A. Joch, F. Kossentini, and G.J. Sullivan, "Rate-constrained coder control and comparison of video coding standards," IEEE trans. on Circ. Syst. Vid. Techn., vol. 13, no. 7, pp. 688 - 703, 2003. 
[22] S. Ma, W. Gao, and Y. Lu, "Rate-distortion analysis for H.264/AVC video coding and its application to rate control," IEEE trans. on Circ. Syst. Vid. Techn., vol. 15, no. 12, pp. 1533 - 1544, 2005.

[23] H. Wang and S. Kwong, "Rate-distortion optimization of rate control for H.264 with adaptive initial quantization parameter determination," IEEE trans. on Circ. Syst. Vid. Techn., vol. 18, no. 1, pp. $140-144,2008$.

[24] K.-B. Kim and H.-J. Kim, "Back-pressure buffering scheme to improve the cell loss property on the output buffered atm switch," in Proc. IEEE LCN, 1996, pp. $242-248$.

[25] ETSI, "Multimedia Broadcast/Multicast service. (MBMS); UTRAN/GERAN requirements," Tech. Rep., 3GPP TR 25.992-140, 2005.

[26] V. Vukadinovic and J. Huschke, "Statistical multiplexing gains of H.264/AVC video in E-MBMS," in Proc. Int. Symp. on Wirel. Perv. Comp., 2008, pp. $468-474$.

[27] x264 Home Page, "Videolan organization. retrieved 2011-03-11.," 2011.

[28] ffmpeg Documentation, "http://ffmpeg.org/ffmpeg.html," .

[29] J. Yang, Q. Dai, W. Xu, and R. Ding, "A rate control algorithm for MPEG-2 to H.264 real-time transcoding," Proc. IEEE VCIP, vol. 5960, pp. 1995 - 2003, 2005.

[30] S. Ma, v Gao, and Y. Lu, "Rate-distortion analysis for H.264/AVC video coding and its application to rate control," IEEE trans. on Circ. Syst. Vid. Techn., vol. 15, no. 12, pp. 1533 - 1544, 2005.

[31] L. Teixeira and L. Corte-Real, "Coding multiple H.264 video streams using SSIM as quality metric," in Proc. IEEE Int. Workshop on Multimedia Analysis and Processing, 2008, pp. 741 - 748.

[32] K. Deep Singh, A. Ksentini, and B. Marienval, "Quality of experience measurement tool for SVC video coding," in ICC, 2011. 